\title{
A population based study of unintentional firearm fatalities
}

D Cherry, C Runyan, J Butts

\begin{abstract}
Objectives-To describe the circumstances of fatal unintentional firearm injuries in a statewide population in a region of the United States with high firearm fatality rates and to compare to similar data from an earlier period in the same state.

Methods-Analyses of North Carolina medical examiner database (1985-94) and review of medical examiner case reports (1990-94) and comparison to similar data from 1979-82.

Results-A total of 390 unintentional shooting deaths occurred $(0.59 / 100000$ population) between 1985-94 with the highest rate in the ages 15-24. Between 1990-94, handguns were responsible for $59 \%$ of these deaths compared to $40 \%$ in the 1979-82 period. Younger victims were more likely to be shot by family or friends, though, $53 \%$ of all deaths were self inflicted. In 45 cases, the person firing the weapon was reported to believe that the gun was unloaded or had the safety device activated.
\end{abstract}

Conclusions-This study demonstrates changes in patterns of unintentional firearm fatalities in North Carolina in two decades, particularly the increase in incidence of events involving handguns. The results highlight the need for additional attention to efforts governing access to firearms, particularly handguns; technological advances in designing safer guns, and additional emphasis on safe storage policies and practices.

(Injury Prevention 2001;7:62-65)

Keywords: firearms; mortality; trauma

ion, Univers

School of Public

Health and University

of North Carolina

Injury Prevention

Research Center

C Runyan

University of North

Carolina Injury

Prevention Research

Center and North

Carolina Office of the

Chief Medical

Examiner

J Butts

Correspondence to: Dr Carol Runyan, Injury Prevention Research Center, CB 7505 Chase Hall, University of North Carolina, Chapel Hill, NC 27599-7505, USA

carol_runyan@unc.edu

In 1998, firearms were responsible for 30708 fatalities in the United States, accounting for $21 \%$ of all injury deaths. ${ }^{1}$ The majority were homicides and suicides, though unintentional firearm fatalities account for $3 \%$ of the total. ${ }^{1}$ Unintentional firearm fatalities in the United States dropped from 1501 in 1988 to 866 in 1998. ${ }^{1-3}$ However, for every unintentional firearm fatality, an estimated 14-20 non-fatal injuries are treated in United States hospitals and emergency departments, resulting in as many as 20000 injuries annually. ${ }^{34}$

The southern United States, including North Carolina, has one of the highest unintentional firearm death rates (0.52/ 100000 ), compared to rates of 0.13 in the northeast, 0.27 in the west, and 0.32 in the midwest United States. ${ }^{1}$ In 1998, the rate in
North Carolina (0.62) was nearly double that of the national rate $(0.34) .{ }^{1}$ This paper updates earlier research in North Carolina, ${ }^{5}$ describing cases from 1985 through 1994 and highlighting changes in patterns.

\section{Methods}

Using data available from the North Carolina Office of the Chief Medical Examiner (OCME), we selected those cases in which the means of death was a firearm and the manner of death was an "accident". In North Carolina, county medical examiners conduct the original investigation and certify as to cause and manner, deaths occurring under "suspicious, unusual or unnatural circumstances", due to "violence, poisoning, accident, suicide or homicide", or unattended by a physician. ${ }^{6}$ This includes all firearm deaths. Cases are then sent to the OCME where they are reviewed by a forensic pathologist, amended if necessary, and stored for five years before being moved to a central storage location. We obtained data from the OCME electronic database for the full 10 year period and reviewed hard copies of case files for all fatalities during the years 1990-94 to describe the circumstances leading to the fatality, characteristics of the shooter and firearm, and details about the firearm owner. Case files contained a Report of Investigation by Medical Examiner, a copy of the death certificate, and may have included other documents such as an autopsy report, toxicology screen report, case notes, correspondence, and newspaper clippings. Occasionally, but not uniformly, police reports are included in the file.

\section{Results}

A total of 390 unintentional firearm fatalities occurred in North Carolina between the years 1985 and 1994. The mean age for all decedents was 26 , with a range of $0-86$ years. Decedents between the ages of 15 and 24 accounted for $37 \%$ of all cases (1.35/100 000 residents). Males made up a disproportionate share of the decedents $(87 \%)$ and $\mathrm{racial} / \mathrm{ethnic}$ minorities were slightly over-represented $(31 \%$ of decedents $v 25 \%$ of North Carolinians).

Detailed reviews of all case files from 1990-94 ( $n=187)$ revealed that information specifying the shooter's relationship to the decedent was available in $94.5 \%$ of the cases. Fifty three per cent of the fatalities were self inflicted. Decedents between the ages of 5 and 14 were most commonly shot by friends $(35 \%)$, brothers $(24 \%)$, or themselves $(22 \%)$. For all other ages, the fatal wounds were more 
Table 1 Circumstances * of unintentional firearm fatalities: North Carolina, 1990-94 $(n=187)$

\begin{tabular}{ll}
\hline Circumstance category (mutually exclusive) & No (\%) \\
\hline "Playing", "fooling around" & $65(35.0)$ \\
Cleaning, repairing, loading, unloading & $23(12.0)$ \\
"Went off" & $23(12.0)$ \\
Hunting (in the field) & $18(10.0)$ \\
Returning to, removing from storage & $13(7.0)$ \\
Dropped & $9(5.0)$ \\
Demonstrating, transferring & $9(5.0)$ \\
Other & $7(3.5)$ \\
Defective gun, ammunition & $5(3.0)$ \\
Target shooting, firing range & $4(2.0)$ \\
Tripping, falling & $3(1.5)$ \\
Carrying on person & $3(1.5)$ \\
Child picking up gun (<4 years old) & $2(1.0)$ \\
Stated unknown/unspecified & $2(1.0)$ \\
Argument, fight & $1(0.5)$ \\
Total & $187(100)$
\end{tabular}

*Abstracted from case files of all 187 fatalities that occurred during 1990-94, a subset of the 390 total cases that occurred during 1985-94.

likely to be self inflicted. Among the 21 female decedents, seven were shot by a spouse or boyfriend, four shot themselves, four were killed by other family members, and six by various other people. In $43 \%$ of the 88 cases in which the decedent was under age 20 and $50 \%$ of the 16 cases under age 10 , the shooter was described as "playing with the gun".

Circumstances of the shootings are reported in table 1 . These mutually exclusive categories parallel those used in the earlier study by Morrow and Hudson ${ }^{5}$ and describe the activity or event immediately preceding the injury. Incidents were placed in these categories based on available information in the OCME records. A coding hierarchy was established so that any incident occurring in the field during hunting was placed in the hunting category. Incidents that occurred while preparing for or upon returning from a hunting trip were not categorized as hunting.

The decedent was alone in $21 \%$ of all cases and in $38 \%$ of those self inflicted. Decedents age 45 and older were more likely to be alone at the time of the injury (67\%) compared with younger decedents $(20 \%)$. Although state law requires an adult to be present when a child younger than 12 handles a firearm, in only four of the 29 cases involving children under 12 was this the case.

Information in the case narrative about the shooter's belief that the gun was unloaded or had a safety device on was available for 105 of the 187 abstracted cases. This information is based on investigators' interviews with witnesses and/or relatives. The shooter was reported to know that the gun was loaded in only 60 of the 105 cases and to have believed the gun to be unloaded in 39 cases. The safety device was believed to be on in the remaining six cases.

Blood alcohol concentrations (BACs) were determined at the time of autopsy for $90 \%$ of the 390 cases in the 10 year series (1985-94) with positive results recorded for $22 \%$ of those tested. All who tested positive were between the ages of 15 and 64 . The average BAC was $146 \mathrm{mg} / \mathrm{dl}$, with a range of $20-350 \mathrm{mg} / \mathrm{dl}$; of those with positive tests, $72 \%$ had BACs greater than $100 \mathrm{mg} / \mathrm{dl}$. Thirty two per cent of
Table 2 Firearm types used in unintentional firearm fatalities: North Carolina, 1990-94 $\left(n=187^{\star}\right)$ and 1979-82 $\left(n=149^{\star}\right)$ t; results are number $(\%)$

\begin{tabular}{lll}
\hline Type & $1979-82$ & $1990-94 \ddagger$ \\
\hline Handguns & $59(40.4)$ & $109(59.2)$ \\
$\quad$ Semiautomatic & & $19(10.3)$ \\
$\quad$ Revolver & & $32(17.4)$ \\
$\quad$ Other/unknown handgun & $35(31.5)$ \\
Rifles & $52(35.6)$ & $40(19.0)$ \\
Shotguns & $146(100)$ & $184(100)$ \\
Total & & \\
\hline
\end{tabular}

^The type of firearm was unspecified for three cases in each time period.

†Morrow PL, Hudson P. Accidental firearm fatalities in North Carolina, 1976-80. Am f Public Health 1986;76:1120-3.

$\ddagger$ The percentages do not total $100 \%$ due to rounding.

those who died from self inflicted injuries tested positive for alcohol compared with $7 \%$ of those whose injuries were inflicted by another person.

In all but three cases, the type of firearm was noted in the file (table 2). Handguns were the most common weapons involved (59\%), followed by shotguns and rifles. The caliber of rifles and handguns and the gauge of shotguns was identified for 174 (93\%) of the cases. In addition, two weapons were Derringers (no caliber noted) and one was an M-16 rifle.

Of the 109 handguns, revolvers (29\%) were slightly more common than semi-automatics $(17 \%)$. The mechanism of action was unspecified for 54 handguns $(50 \%)$ and listed as some other type for four (4\%).

Information about gun ownership was unavailable for $30 \%$ of cases. For the 132 cases in which the information was available, $40 \%$ of the guns were reported as owned by the decedent and $60 \%$ by other persons, usually a family member.

Nearly $68 \%$ of all deaths occurred between September and March with the most fatalities recorded in November (14\%) and December $(13 \%)$. Of the hunting fatalities, $89 \%$ occurred between October and December. These cases were more likely to involve shotguns and rifles than shootings at other times of the year.

\section{Discussion}

Consistent with earlier studies, ${ }^{2589}$ we found that children and teenagers continue to be over-represented as victims in unintentional shootings. Although adults were less likely to find a loaded firearm and "play" with it, acts as simple as removing a loaded firearm from storage proved deadly for some.

It is notable that the number of fatalities is greater during the fall, even excluding in-thefield hunting incidents. This most likely is the consequence of increased use and handling associated with guns in anticipation of hunting or during transport to and from hunting.

The patterns have changed since 1980 . Although earlier data demonstrated that most decedents had been shot by others, ${ }^{5}$ usually with shotguns, we found that most people had shot themselves, usually with handguns. Overall, the proportion of handguns involved in unintentional shootings increased by $51 \%$ (from $39 \%$ to $59 \%$ ) since the earlier report. ${ }^{5}$ 
Also, in the later period, injuries inflicted by others were more likely to be friends than family members; a pattern different from that described by Morrow and Hudson. ${ }^{5}$ A recent study in Georgia indicated that $35 \%$ of households reported owning guns, including at least one handgun in $69 \%$ of the households. ${ }^{10}$ This compares to figures reported by Cook indicating that approximately $50 \%$ of all households nationally owned guns in 1959 and 1979, but the proportion of gun owning households possessing handguns increased from about a quarter in 1959 to half in $1979 .{ }^{11}$ Consequently, it is not clear how much gun ownership patterns as opposed to other issues have contributed to the increased involvement of handguns in North Carolina unintentional shootings since 1980 .

Although a standard investigation form is used by the local medical examiners, the reported details vary, particularly for factors not essential to determining the manner and means of death. Little information is available about the firearm manufacturer, model, mechanism of action, modifications or defects, and date of purchase, making it hard to identify specific products for recall, stopping production, or design modification. Detailed information about the shooter is also scarce in medical examiner records. Because not all cases are self inflicted, it is important to know about the other person involved when developing interventions.

Misclassification by the medical examiners of the manner of death as "accident" when it might actually have been "suicide" or "homicide" is a potential problem. However, all firearm death investigations at the county level undergo a secondary review at the OCME where a plausible explanation for the designation of "accident" must be present before it can be accepted as the final manner of death. In addition, further investigation of the incident may be conducted by the OCME. Though misclassification can certainly occur, the level of review at the OCME and the use of standard classification procedures minimizes this possibility.

This study confirms earlier evidence describing the problem of unintentional firearm fatalities and the relatively small change in incidence over the past 20 years despite increasing public awareness of both unintentional and intentional firearm injuries. Future research should examine non-fatal firearm events, which greatly outnumber deaths, ${ }^{34}$ and should attempt to collect data to assess the effectiveness of designing safer firearms, ${ }^{12}{ }^{13}$ modifying the characteristics and availability of ammunition, ${ }^{12}{ }^{14}$ imposing and enforcing legal restrictions on sales and purchases of guns, ${ }^{14}$ and making firearm owners liable for injuries resulting from use of their weapons. ${ }^{14}$

\section{Implications for prevention}

In the meantime, the problem requires preventive action through both policies and supporting educational interventions that consider all

\section{Key points}

- The US experienced close to 900 fatal and 20000 non-fatal unintentional shootings in 1998.

- $59 \%$ of the 390 North Carolina deaths between 1985 and 1994 were associated with handguns, a $51 \%$ increase over 10 years, corresponding to increased ownership of handguns.

- Many victims believed the gun was unloaded; more than one third of deaths were caused by playing with loaded weapons.

- More than $80 \%$ of children were killed by friends and relatives; most adult deaths were self inflicted.

- Misclassification of suicides is possible, but case review procedures mitigate against this.

- Interventions are urged to address gun design, ownership, and storage.

elements of the Haddon model and the multiple dimensions of intervention decision making. ${ }^{15}$ In this vein, interventions could address gun owners' behaviors (for example, purchasing practices, use patterns, storage behaviors) and might include special warning messages broadcast at the start of hunting season; improvements in product design so as to reduce the hazard potential of the gun; modifications in the physical environment through improved facilities for storing guns either within or outside the home; and by addressing home security issues that relate to decisions to acquire handguns for protection. Also, alterations in social environments that reduce ownership and, for gun owning households, foster safer usage (for example, removal of firearms from homes with children, legal accountability for unsafe access, or role modeling through inclusion of material in popular media programs that demonstrate safety promoting decisions). Interventions such as these should be carefully evaluated to determine effects not only on unintentional shootings, but also homicide and suicide.

This work was partially supported by a grant to the University of North Carolina Injury Prevention Research Center from the National Center for Injury Prevention and Control (R49/ CCR402444). The authors acknowledge the assistance of Elizabeth Hooten in accessing the data in the Office of the Chief Medical Examiner and Drs Laura Sadowski and Ronda Zakocs for their comments on a prior draft of the paper.

Ms Cherry now works at the Colorado Department of Public Health and Environment.

1 Office of Statistics and Programming, National Center for Injury Prevention and Control, Centers for Disease Control and Prevention. www.cdc.gov/ncipc/wisqars

2 Baker SP, O’Neill B, Ginsburg MJ, et al. The injury fact book. 2nd Ed. New York: Oxford University Press, 1992.

3 Annest JL, Mercy JA, Gibson D, et al. National estimates of nonfatal firearm-related injuries: beyond the tip of the iceberg. ₹AMA 1995;273:1749-54

4 Lee RK, Waxweiler RJ, Dobbins JG, et al. Incidence rates of firearm injuries in Galveston, Texas, 1979-1981. Am f Epi-

5 Morrow PL, Hudson P. Accidental firearm fatalities in North Carolina, 1976-1980. Am f Public Health 1986;76: $1120-3$. 
6 North Carolina General Statutes $\$ 130$ A-383.

7 North Carolina General Statutes \$ 14-316.

8 Alexander GR, Massey RM, Gibbs T, et al. Firearm-related fatalities: an epidemiologic assessment of violent death. $A m$ f Public Health 1985;75:165-8.

9 Cole TB, Patetta MJ. Hunting firearm injuries, North Carolina. Am f Public Health 1988;78:1585-6.

10 Kellermann AL, Fuqua-Whitley DS, Sampson TR, et al. Public opinion about guns in the home. Inj Prev 2000;6:189-94

11 Cook PJ. The influence of gun availability on violent crime patterns. In: Tonry M, Morris N, eds. Crime and justice: an annotated review of research. Vol 4. Chicago: University of Chicago Press, 1983: 49-89.

12 Kellermann AL Preventing firearm injuries: a review of epidemiologic research. Am $f$ Prev Med 1993;9(suppl demiologic

13 Wintemute GJ, Kraus JF, Teret SP, et al. Unintentional firearm deaths in California. F Trauma 1989;29:457-61.

14 Christoffel KK. Toward reducing pediatric injuries from firearms: charting a legislative and regulatory course. Pediatrics 1991;88:294-305.

15 Runyan CW. Using the Haddon matrix: introducing the third dimension. Inj Prev 1998;4:302-7.

\section{Rib tickler}

The Australian newspaper (18 September 2000) reported that international English cricketer, Alan Mullally, has been ruled out of the cricket tour of Kenya and Pakistan because of injury. Mullally had a sneezing fit which fractured a rib and will be out of action for some time.

\section{Transport policy with bells on}

Britain's deputy prime minister, John Prescott, has revealed his secret plan for getting the country on the move after a period of transport chaos, while at the same time improving road safety. He has announced that all new bikes will have to be sold with bells on them! Safety lobbies have welcomed the announcement, with the Pedestrians' Association calling for action against the "Lycra louts" who ride on the pavement at high speeds. At least, now we will be able to hear them coming.

Pokémon hazard

A 4 year old Turkish boy jumped from a seventh floor balcony copying the characters in the Japanese TV cartoon series Pokémon. He landed on soil, suffering a broken leg. He continues to be a fan of Pokémon, despite his injury (Daily Telegraph (London), October 2000). 